Research Article

\title{
Novel PIFA with Minimum Height and Maximum Gain for GSM Receiving Band
}

\author{
Y.J.Nazeer Ahmed* and S.Kaja Mohideen \\ ECE Department,B.S.Abdur Rahman University, Chennai 600048,India.
}

Received 3 March 2016; Accepted 4 June 2016

\begin{abstract}
A simple and novel Planar inverted F antenna (PIFA) with minimum height and enhanced gain has been designed suitable for GSM Receiving band. The designed PIFA covers the frequency range of $900-960 \mathrm{MHz}$ and it has minimum height of $5 \mathrm{~mm}$ from ground to top of the patch. The proposed antenna structure has been simulated using HFSS and then fabricated using FR4 epoxy material. The designed antenna has better return loss and good impedance matching to its credit.
\end{abstract}

Keywords: PIFA antennas, GSM Receiving Band, Gain.

\section{Introduction}

Planar Inverted FAntenna is very compact in size while compared with other microstrip antennas. It consists of a radiating patch, ground plane and shorting pin or plate to connect radiating patch with ground plate. pifa antenna will resonate at a size of quarter wavelength of operating frequency while compared with other type of microstrip antennas which require half of its wavelength. Due to the above reasons, the size of the PIFA antenna is reduced to half compared with other type of antenna. Due to its compact size and portability, it is widely used in cell phone as a transmitting and receiving antenna. One of the best advantage of PIFA is that, it has very low backward radiation which makes it a preferable choice in cell phone to avoid health hazards for the mobile phone users. It has omnidirectional radiation pattern. The shorting plate or pin and feeding point are responsible for matching of impedance. The antenna bandwidth can be optimized by varying the size of the ground plane.[1]-[3].

The main drawback of existing mobile phone antenna is their height that has an influence on the size of the mobile phone. This has been taken care of in the proposed antenna design. The main objective of this work is to design a high gain PIFA with minimum height to suit GSM receiving band. By fixing antenna height as $5 \mathrm{~mm}$ the structure of PIFA has been modified to obtain resonant frequency at GSM receiving band. The feeding point and shorting pin plays a major role in obtaining a maximum gain and better impedance match[4].

\section{Conventional Antenna Geometry}

\footnotetext{
*E-mail address: yjnazeer@gmail.com ISSN: 1791-2377 @ 2016 Eastern Macedonia and Thrace Institute of Technology.All rights reserved.
}

The conventional PIFA antenna which has ground plane, radiating patch, shorting plate and feeding point was shown in fig..1.The resonant frequency of PIFA is a quarter wavelength of its operating frequency. The size of radiating patch $(\mathrm{L} 1+\mathrm{L} 2)$ and the width of shorting plate $\mathrm{W}$ will be responsible to obtain the resonant frequency. The resonant frequency can be obtain using the following equations:

$\mathrm{L} 1+\mathrm{L} 2=\lambda / 4$

where L1 and L2 are Length and width of radiating patch. when

$\mathrm{W} / \mathrm{L} 1=1$ then $\mathrm{L} 1+\mathrm{H}=\lambda / 4$.

whereas if

$\mathrm{W}=0$ then $\mathrm{L} 1+\mathrm{L} 2+\mathrm{H}=\lambda / 4$

where $\mathrm{W}$ and $\mathrm{H}$ are the width and height of the shorting plate respectively.

\section{Proposed Antenna}

The proposed antenna has been designed using Epoxy FR4 substrate which can be available easily at reasonable cost and its relative dielectric constant value $\mathrm{er}$ is 4.4

\section{The Dimensions of Proposed Antenna}

The radiating patch and ground plane of the proposed antenna which is one side copper coated Fr4 Epoxy of thickness 1.6 and dielectric constant 4.4 is shown below. In radiating patch copper should face outwards (above FR4) were as in ground plane the copper should face downwards (below FR4) direction. 


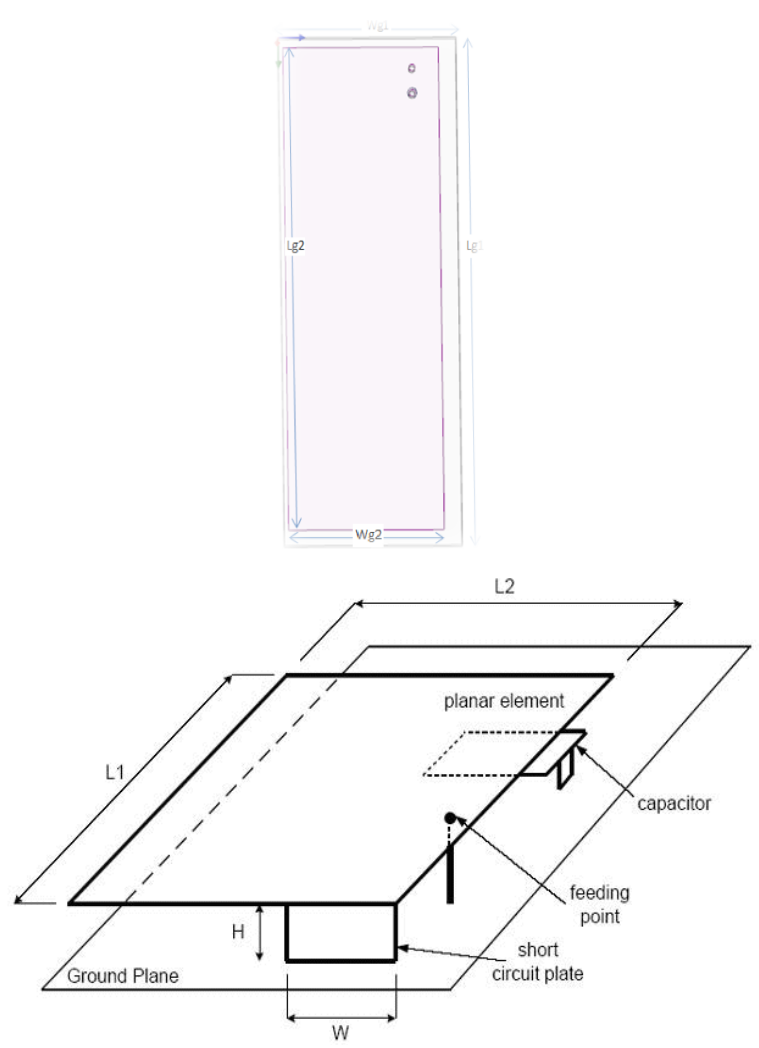

Fig.1.PIFA Antenna

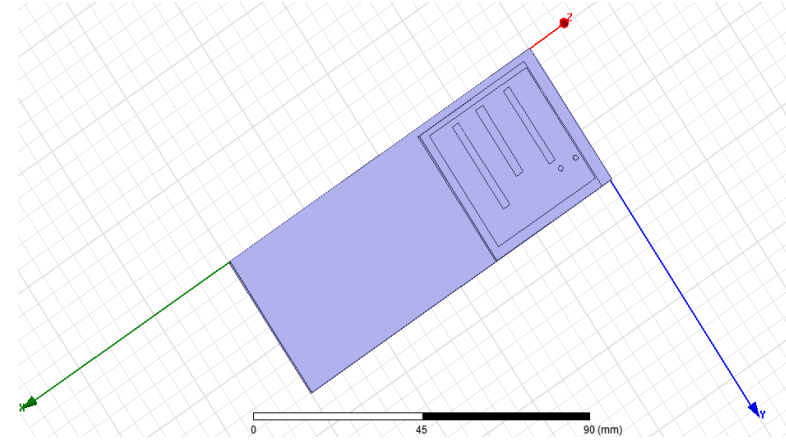

Fig. 2. Top View of proposed antenna

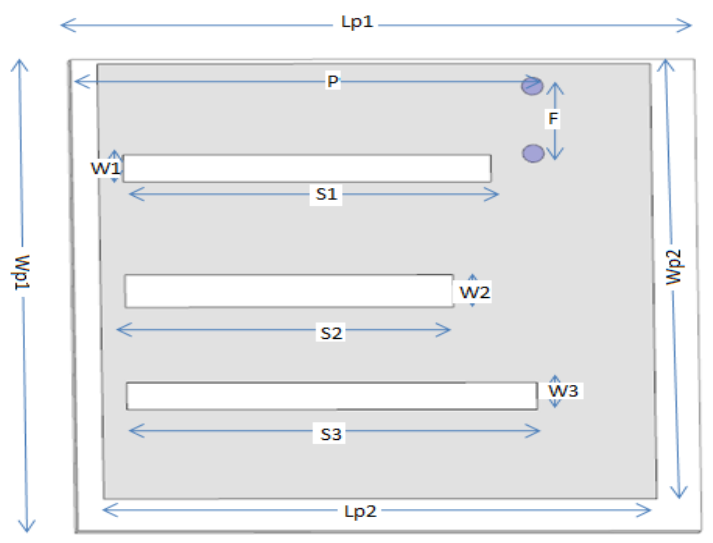

Table1. Antenna parameters dimensions.

\begin{tabular}{l|l|l|l|l|l|l|l|l|l}
\hline Parameters & Lp1 & Lp2 & Wp1 & Wp2 & P & F & W1 & W2 & W3 \\
\hline In $(\mathrm{mm})$ & 38 & 33.7 & 35 & 32.3 & 30 & 5 & 2 & 2.4 & 2 \\
Parameters & S1 & S2 & S3 & Lg1 & Lg2 & Wg1 & Wg2 & H & \\
In $(\mathrm{mm})$ & 22.4 & 20 & 25 & 100 & 95 & 40 & 35 & 5 & \\
\hline
\end{tabular}

The coordinates for ( $\operatorname{Lg} 1, \mathrm{Wg} 1),(\mathrm{Lg} 2, \mathrm{Wg} 2)$,
( Lp1,Wp1), (Lp2,Wp2), (Lp2,Wp2), P, F are $(0,0,0),(2,1,0),(4,1.9,5),(4.33,3.5,5)$, $(6,30,5),(11,30,5)$ respectively.

The resonant frequency length $\lambda / 4$ for GSM receiving band is approximately $84 \mathrm{~mm}$. As per the equations 1 to 3 given above, the length of $84 \mathrm{~mm}$ includes the dimension of $\mathrm{L} 1, \mathrm{~L} 2$ and $\mathrm{W}$. In the proposed antenna, slots in the radiating patch were introduced, which increased the electrical length required to resonate the GSM band and at the same time reduced the size of the radiating patch to $66 \mathrm{~mm}$. The width of the shorting pin is $1.3 \mathrm{~mm}$. The ground plane of proposed antenna is $95 \mathrm{mmx} 35 \mathrm{~mm}$. The total height of the antenna from ground to top of the patch is $5 \mathrm{~mm}$. The thickness of FR4 used in this proposed antenna is $1.6 \mathrm{~mm}$. Hence the air gap between the ground plane and radiating patch is very much reduced to $2.8 \mathrm{~mm}$. The top and side view of the proposed antenna is shown in fig. $2 \& 3$. In proposed design the position of feeding point and shorting pin were finalized accordingly to acheive better result as mention in table1.
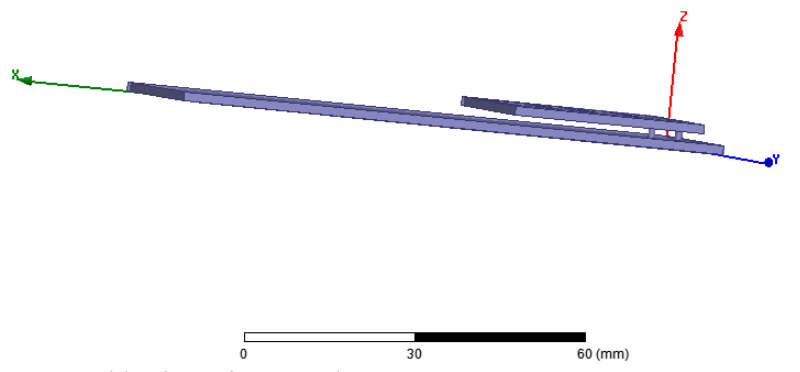

Fig. 3. Side view of proposed antenna

The top and Side view of Fabricated antenna for the above dimension is shown in fig. 4,5.

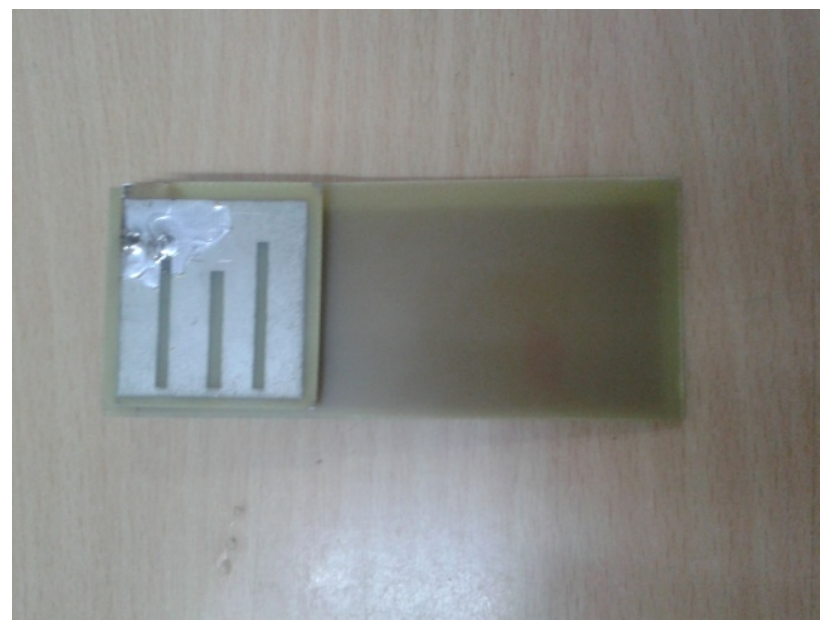

Fig. 4. Top View

\section{Simulation Results}

The simulation and optimization of the antenna has been performed by using High Frequency Structure Simulator (HFSS) of version 13. The return loss of the antenna is taken as a vital parameter since it gives the information about the operating frequency at which the designed antenna is resonating. The value of Return Loss should be $-6 \mathrm{~dB}$ as a marginal level[11]. If it is less than $-6 \mathrm{~dB}$, then it will improve the antenna performance. The proposed antenna geometry on simulation yielded the Return loss of $-6 \mathrm{~dB}$ for the frequency range of $906 \mathrm{MHz}$ to $945 \mathrm{MHz}$. Fig. 6 shows the simulated return loss of proposed antenna. 


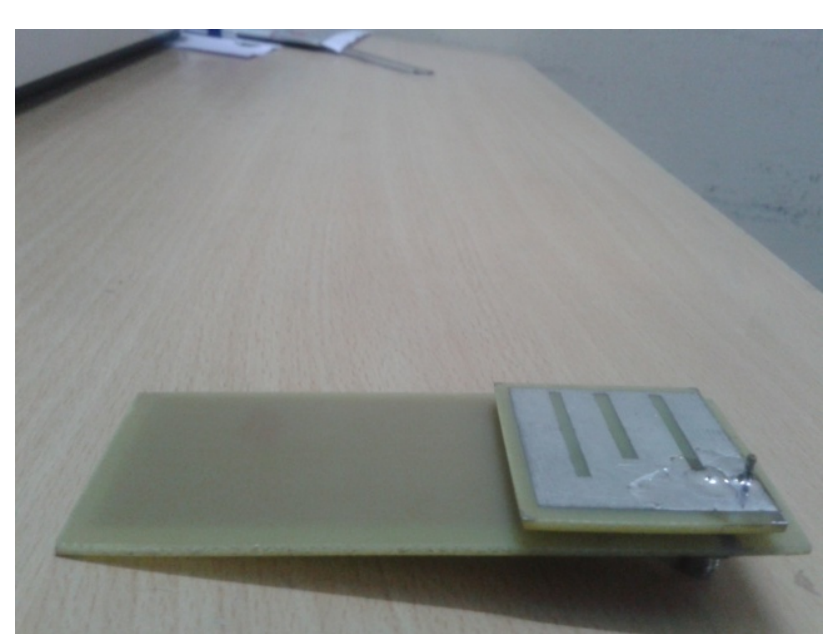

Fig. 5. Side View

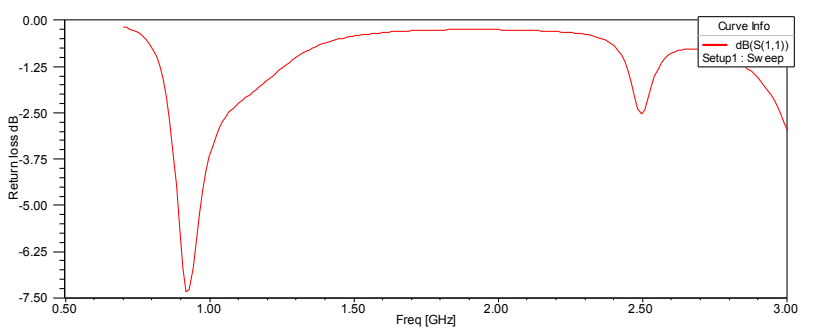

Fig. 6. Return loss

The next important parameter to be considered is impedance matching since it results in maximum power transfer .The antenna impedance should be $50 \mathrm{ohms}$ in general so that to integrate with other part of the circuit in a system without any power loss. The simulation results in fig. 7 shows that impedance of proposed antenna for resonant frequency of $921 \mathrm{MHz}$ is $57.15 \mathrm{Ohms}$ which is much closer to the ideal value of $50 \mathrm{Ohms}$.

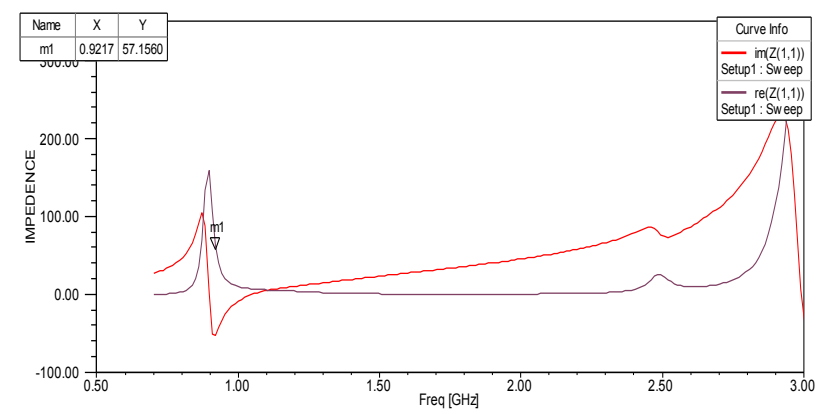

Fig. 7. Impedence plot of proposed antenna.

The third parameter of antenna which is to be considered is radiation pattern through which the gain of the antenna at a particular operating frequency can be obtained. The simulated antenna radiation pattern is shown in fig. 8 .

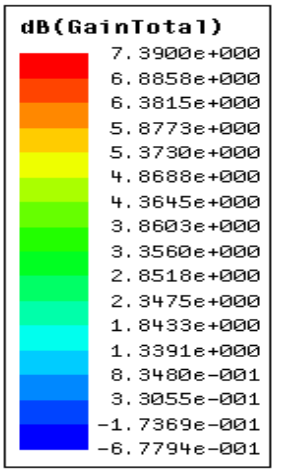

Fig. 8. Radiation pattern of proposed antenna

The simulation result shows that a maximum gain of 7.5 $\mathrm{dB}$ can be achieved using the proposed structure of antenna .

\section{Results and Discussions}

\section{Return Loss:}

The Return loss of proposed antenna has been measured by using Agilent Vector Network Analyzer (N9925A). From fig. 9 it can be viewed that, in the frequency range of 886 $\mathrm{MHz}$ to $960 \mathrm{MHz}$ the proposed antenna yielded Return loss of $-6 \mathrm{~dB}$, and a value of $-9.21 \mathrm{~dB}$ for the frequency of 919.8 $\mathrm{MHz}$ which is better than the simulated value.
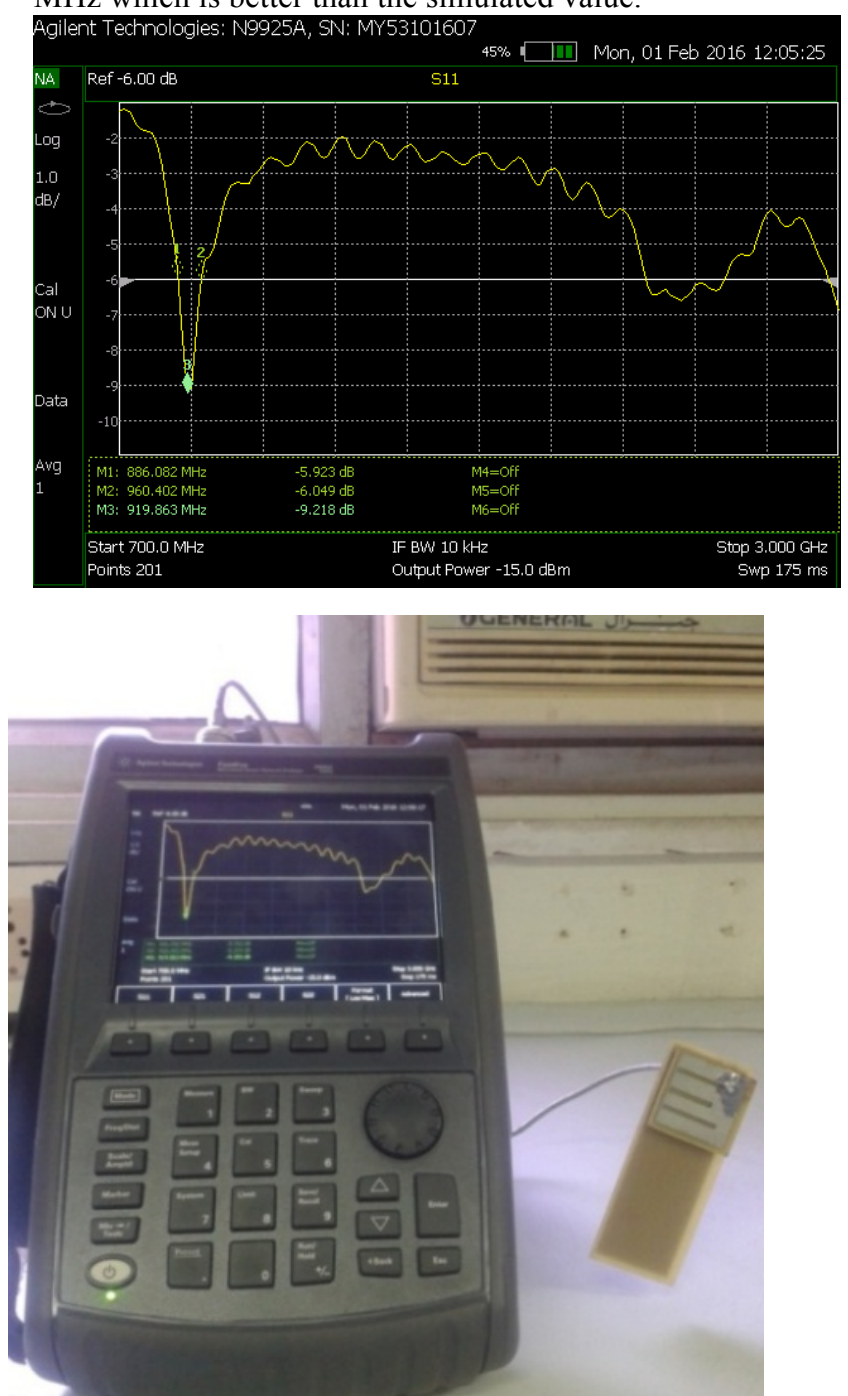


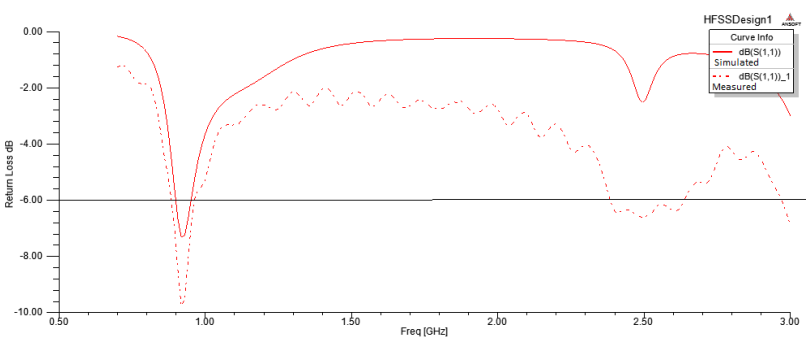

Fig. 9. Measured and simulated Return loss.

\section{Smith chart:}

Similarly the smith chart of the proposed antenna has been measured using VNA as given in fig. 10. At the frequency of 921.6 $\mathrm{MHz}$ and 936.2 $\mathrm{MHz}$, the impedance of $53 \Omega$ and $52 \Omega$ were obtained,which is much near to the ideal value of $50 \Omega$.

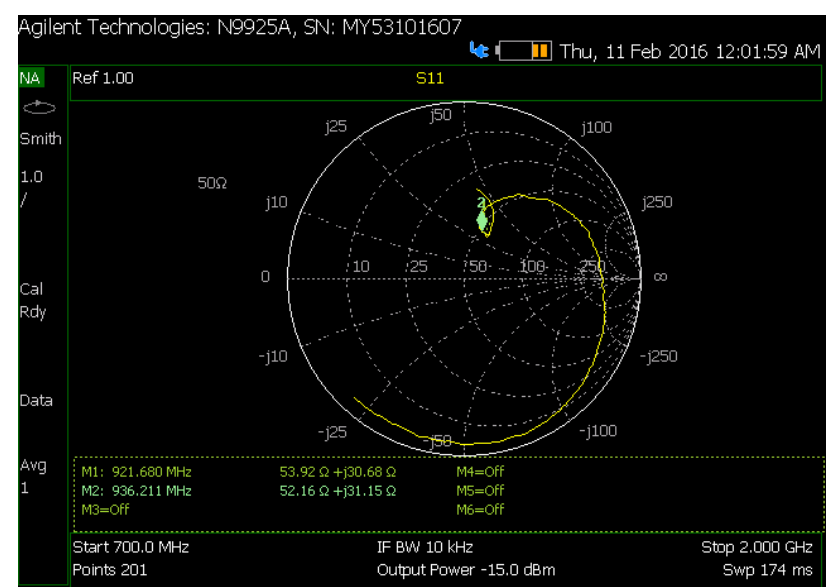

Fig. 10. Measured Smith Chart.

\section{Radiation pattern:}

The Radiation pattern of the proposed antenna has been measured using Anechoic Chamber of name SATIMO as displayed in fig. 11. At the resonating frequency of 920 $\mathrm{MHz}$, simulated and measured radiation pattern for phi $=0$ and phi $=90$ has been obtained as shown in fig. $12 \& 13$. The measured gain varies from 1.5 to $2 \mathrm{~dB}$ for frequency band of 900 to 960 with a interval $10 \mathrm{MHz}$ each. A Maximum value of $7.5 \mathrm{~dB}$ is obtained form simulation at the frequency of $935.535 \mathrm{Mhz}$.

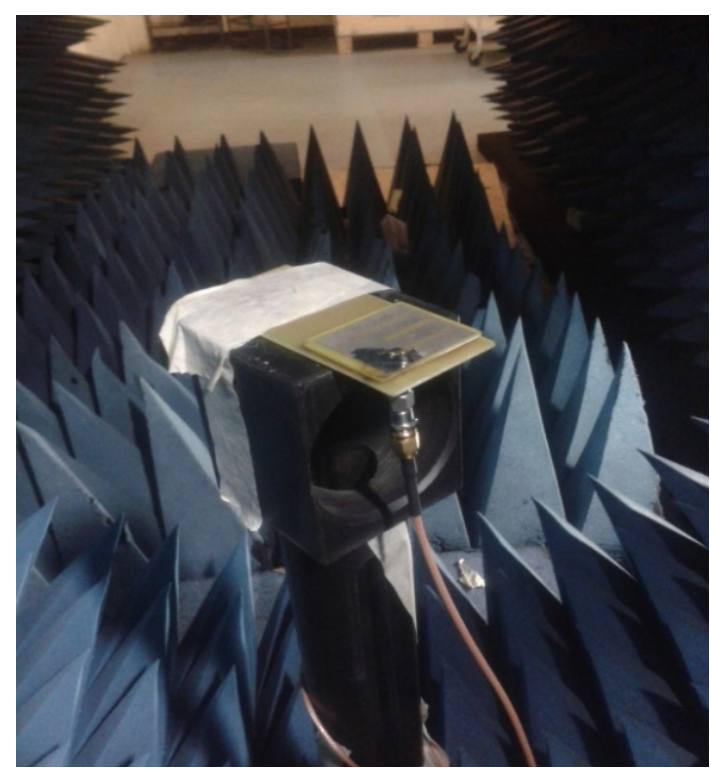

Fig. 11. Proposed antenna in Anechoic chamber for gain measurement.

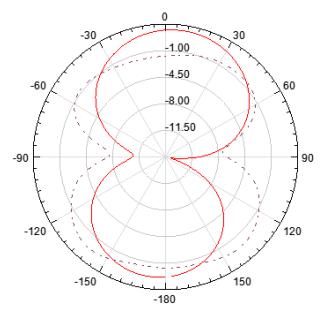

Fig. 12: Radiation pattern for $920 \mathrm{Mhz}$ at $\mathrm{Phi}=0$.
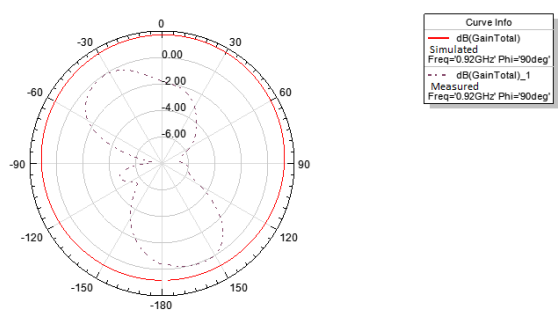

Fig.13. Radiation pattern for $920 \mathrm{Mhz}$ at $\mathrm{Phi}=90$.

On comparing the parameters of the proposed PIFA antenna with that of differential antenna designed by Mahima Arrawatia et al[12], it can be seen that the proposed antenna has as gain much closer to it but achieved with a height of $5 \mathrm{~mm}$ as indicated in table 2 .

Table 2. Comparison of results.

\begin{tabular}{l|l|l}
\hline Parameters & $\begin{array}{l}\text { Differential } \\
\text { antenna }\end{array}$ & proposed \\
\hline Antenna type & Differential & PIFA \\
Radiating patch & $82 \times 82 \mathrm{~mm}^{2}$ & $33.7 \times 32.3$ \\
Dimensions & & $\mathrm{mm}^{2}$ \\
Gain & $8.5 \mathrm{~dB}$ & $7.5 \mathrm{~dB}$ \\
Antenna Height & $18 \mathrm{~mm}$ & $5 \mathrm{~mm}$ \\
\hline
\end{tabular}

\section{Conclusion}

A novel PIFA antenna with minimum height of $5 \mathrm{~mm}$ is proposed and fabricated. The fabricated antenna resonates at $919.8 \mathrm{MHz}$ of GSM band with a return loss of $-9.21 \mathrm{~dB}$. It yielded the impedence of $53 \Omega$ and $52 \Omega$ at $921.6 \mathrm{MHz}$ and 936.2 $\mathrm{MHz}$ respectively. A maximum gain of $7.5 \mathrm{~dB}$ is achieved in the proposed simulated antenna by fixing the position of feeding point and shorting pin accordingly. The proposed PIFA antenna have better performance while compared with the existing differential antenna. 


\section{References}

1. Kin-Lu Wong, "Planar Antennas for Wireless Communication", Published by John Wiley \& Sons, Inc.,Chapter: 2, Pages: 26-65, 2003.

2. R. Vaughan, "Model and results for single mode PIFA antenna", IEEE Antennas and Propagation Society International Symposium, Vol. 4, Page(s): 4028 - 4031, 2004.

3. W. Geyi, Q. Rao, S. Ali, and D. Wang, "Handset Antenna Design: Practice and Theory", Progress in Electromagnetic Research Journal (PIER), Vol. 80, Pages: 123-160, 2008.

4. Taeho Son, "Feeding point determination for PIFA type mobile phone handset internal antenna", IEEE Antennas and Propagation Society International Symposium, Vol. 1A, Page(s): 475 - 478, 2005

5. C. Rowell, E.Y. Lam, "Mobile-phone antenna design", IEEE Antennas and Propagation Magazine, Vol.54, No. 4, Page(s): $14-$ 34, 2012.

6. J.A. Ray, S.R.B. Chaudhuri, "A review of PIFA technology", IEEE Indian Antenna week (IAW),Page(s): 1 - 4, 2011.

7. Hang Wong, Kwai-Man Luk, Chi Hou Chan, Quan Xue, Kwok Kan So, Hau Wah Lai, "Small antennas in Wireless Communications", Proceedings of the IEEE Journal, Vol. 100, No. 7, Page(s): 2109 2121,2012.

8. Y. Belhadef, N. Boukli Hacene, "PIFAs antennas design for mobile communications", 7th IEEE International Workshop on Systems, Signal Processing and their Applications, Page(s): $119-122$, 2011.

9. Liping Han, Wenmei Zhang, Jianhong Zuo, Jing Li, "Novel Differential Dual Frequency Antenna for Wireless Comm unication, Microwave Conference, China-Japan Joint,pp.154156,2008 .

10. Hassan Tariq Chattha, Yi Huang, Xu Zhu, and Yang Lu, "An empirical equation for predicting the resonant frequency of planar inverted-F antennas", IEEE Antennas and Wireless Propagation Letters,Vol.8, Page(s): 856 - 860, 2009.

11. Rashid Ahmad Bhatti, Ngoc-Anh Nguyen, Viet-Anh Nguyen and Seong ook Park, "Design of a CompactInternal Antenna for MultiBand Personal Communication Handsets", IEEE Proceedings of Asia-PacificMicrowave Conference, Page(s):1-4, 2007.

12. Mahima Arrawatia, Maryam Shojaei Baghini, Senior Member, IEEE, and Girish Kumar "Differential Microstrip Antenna for RF Energy Harvesting”, IEEE Antennas and Propagation, vol. 63, no. 4, pp.1581-1588,2015. 